\title{
Bronchoscopy: A not-so-innocent invasive examination
}

\author{
Dr. Christos Beretas* \\ *PhD Candidate in Cyber Security at Innovative Knowledge Institute, Paris, France.
}

${ }^{\star}$ Corresponding Author: Dr. Christos Beretas, PhD Candidate (full scholarship) in Cyber Security at Innovative Knowledge Institute, Paris, France; Tel: (+30) 693-8909477; Email: cberetas@ikinstitute.org

Received: February 17, 2019; Accepted: February 18, 2019; Published: February 25, 2020

\begin{abstract}
Bronchoscopy has for many years been the only invasive examination used to screen for bronchus in real time, remove objects that have entered the airway, healing properties, and finally a sample including missing tissue for biopsy for the right follow up medical evaluation and treatment. The usual examination is performed by a flexible bronchoscope which does not cause much discomfort to the patient and causes few complications, complications such as bleeding, cardiac arrhythmias, fever, pneumonia, death, introduction and exacerbation of infection, etc.

This short research is about to overturn this nice and enjoyable environment that has been created regarding the safety of this invasive examination that will eventually and happily very soon be replaced. The present short research is here to overthrow the benefits of bronchoscopy and present the reality as this has not been presented to patients before undergoing an invasive examination that may not eventually need to be done.
\end{abstract}

Dedication: This short research is dedicated to my Father Panagiotis, who recently died.

Keywords: bronchoscopy, hemoptysis, death, complications, pneumonia

\section{Introduction}

Very easily, mainly after a CT scan, a PET/CT has appeared in our lives to improve the quality of imaging data, also including the case but not always the lack of knowledge, recommend that patients undergo an invasive bronchoscopy. that the diagnostic process in short time will end, and the patient is transferred to the next stage of treatment, but if the patient never reaches the treatment stage and is instead hospitalized with complications? according to the literature, it is reported that bronchoscopy is performed by an experienced physician, how can the patient know if the physician is experienced or not?

How can the patient know if during the examination they will not contribute to the examination and trainees which is prohibitive in certain coexisting lung diseases where particular experience is required, such as a patient with bronchectasis, COPD, tec, who they need bronchoscopy from a very experienced physician. Is the procedure is followed of informing the patient prior to bronchoscopy about the pros and cons of the examination as well as their complications? Finally, is it worth doing a bronchoscopy which, depending on the clinical picture of the patient, may eventually have the opposite effect? bronchoscopy is not for everyone.

\section{Analysis}

Bronchoscopy is an invasive examination performed in public and private hospitals as well as in private diagnostic centers and clinics. Before conducting the examination blood tests are performed, including blood and cardiology tests, so, how many tests for a diagnostic examination that is considered safe? The complications that it presents contradict the advantages, advantages that in cases depending on the severity of the patient are disadvantages.

Advantages that become disadvantages when one of the following reasons are met.

1. Inexperienced Invasive physician

2. Bronchoscopy by trainees (depends on case).

3. Inexperienced staff.

4. Hygiene conditions.

5. Disinfection, cleaning, and sterilization of a bronchoscope.

6. The clinical picture of the patient.

7. Consideration of medical examinations to avoid bronchoscopy.

8. Inform the patient about the complications of bronchoscopy.

When one or more of the above is not observed, then the patient is at high risk, as the complications of the bronchoscopy examination can cause the patient even more serious damage than the disease itself led the patient to bronchoscopy. What safe examination are they talking about?

Is there a mortality rate of about $0.5 \%$ these patients were aware of the complications? Should they have done bronchoscopy? have the hygiene rules been adhered to or fallen into a category of the above 8 listed? Although international literature indicates guidelines for safe bronchoscopy examinations, does the patient know whether the guidelines are being followed?

One of the major complications of bronchoscopy is bleeding, ranges for example from low $10 \mathrm{ml}$ to $150 \mathrm{ml}$ which is very high. It is 
a serious complication that needs to be addressed immediately since its origin is complex, it can come from many areas such as pulmonary capillaries, pulmonary arteries, pulmonary veins, and finally large thoracic vessels. Bleeding is directly related to the biopsy, in most cases bronchoscopy is performed to obtain a biopsy, an experienced physician in a burdensome patient with a history who knows before, noted here the bronchoscopy requires the patient to hold already a $\mathrm{CT}$, which will require a CT scan. is the one who will judge it must be done, if it is to be done, to pay attention, what requires observation, coexisted, possible complications, time of examination in relation to the severity of the patient, the participation of trainees or not, and how to get a sample.

There are two types of sample deficiencies: endobronchial and transobronchial biopsy, transobronchial biopsy causes the patient and most bleeding, here is a big question, in patients with bronchiectasis, or similar medical cases that usually occur in these medical cases colonies and bacteria such as pseudomonas aeruginosa that are usually resistant to antibiotics, a transobronchial biopsy is responsible for burdening the patient's clinical picture? Thus, correlated the bleeding with the number of biopsies and how they were obtained. Considering the fact that patients with congenital diseases, medicines that are taken for various important reasons, a bleeding can reach or exceed $40 \%$ depending on the case of patients undergoing bronchoscopy. So why should they do a bronchoscopy? The treating physician should be able to judge the overall picture of the patient in order to be able to make a correct diagnosis, taking into account patients with a poor medical history considering the negative aspects of a bronchoscopy, however, bronchoscopy is an invasive risk examination depending on the patient's physical condition and medical history. Bronchoscopy examination is not for everyone.

It is concluded that the bleeding of the patient after bronchoscopy implies with further clinical progress, although the incidence of sudden death is small as has been mentioned above. Small bleeding can be easily treated large bleeding to stop may require laser and even surgery, the patient must consider all options and exhaust all parameters. It is worth considering whether it is worth the effort, pain and risk to perform a bronchoscopy examination that no one can guarantee his or her physical situation after the examination is completed.

Most complications that have occurred during the examination or thereafter have to do with how to obtain and receive biopsy material. Such cases are brushes that have broken down or have not worked properly. Continuous aspiration during the examination may lead to hypoxemia and atelectasis. Pseudomonas aeruginosa is the most frequently transmitted organism from an inappropriate bronchoscope (see above 8 reasons) which has not been properly disinfected and cannot be used. Using such a bronchoscope is a criminal act against the patient. During bronchoscopy, patients have been observed to have increased blood pressure and increased heart rate, this is due to increased oxygen demand from the myocardium which may then lead the patient to arrhythmias and ischemia. Cardiac arrhythmias that occur after bronchoscopy confess hypoxemia. Particularly in COPD patients with hypercapnia hypoxia is contraindicated for flexible bronchoscopy. It is worth noting at this point that complications of bronchoscopy often occur when there are concomitant diseases such as pneumonia or tumor, as well as the type of forceps to be used, that is toothed or non-serrated forceps. In patients with chronic bronchiectasis, the physician should consider all indications for the overall picture of the patient and whether or not to perform the bronchoscopy. It is the physician that will analyze the patient's respiratory function and the degree of criticality. It should also be borne in mind that some patients with infections will need antibiotics after bronchoscopy, these patients likely to need antibiotics that will be able to deal with germs and bacteria that are resistant. These patients are more likely to develop febrile episodes as well as critical respiratory function.

The physician is solely responsible for analyzing the pros and cons, it is the one who weighs the benefit of the risk. Patients with bronchiectasis should not be bronchoscopied by trainees or by physicians who rarely perform bronchoscopy. When these patients show haemoptysis, the detection of bleeding is crucial. Finally, bronchoscopy in COPD patients is particularly burdensome compared to non-COPD patients, COPD patients should be evaluated on the findings of their clinical trials together with medical evacuation before performing the bronchoscopy. Assessment of potential advantages over advantages must be evaluated.

\section{Conclusion}

Bronchoscopy is a conditional safe examination when all safety rules are met, medical evaluation is performed separately for each patient, each patient is different. Bronchoscopy is not for all patients, generally described as a safe invasive examination but with so many complications that there are people who have had a bronchoscopy and have not returned home, is this safe medical examination they say? it is not a safe medical examination, it is a safe conditional examination, evaluating the clinical picture of the patient in combination with the degree of experience of the physician and the clinical picture of the patient judging its effectiveness.

Patients with co-existing health problems should be assessed for the advantages of screening in conjunction with screening complications. Physicians should not be dragged into the altar of the rapid effect of detecting the problem by undergoing bronchoscopy in patients who are not allowed to do a bronchoscopy based on physical or medical pre-existing health conditions. It is a medical examination with uncertain complications sometimes simple, sometimes heavy and sometimes deadly.

Classical bronchoscopy has begun to come to a second myrrh as soon as virtual bronchoscopy is implemented and thus the complications of classical bronchoscopy will be eliminated. Bronchoscopy should be chosen as a medical examination after all other medical examinations and methods have been utilized and under conditions based on the clinical picture of the patient, in strict compliance with the hygiene guidelines.

\section{References}

1. Kovaleva J, Peters FT, van der Mei HC, Degener JE (2013) Transmission of infection by flexible gastrointestinal endoscopy and bronchoscopy. Clin Microbiol Rev 26: 231-254. [Crossref] 
Dr. Christos Beretas (2020) Bronchoscopy: A not-so-innocent invasive examination

2. Pereira W Jr, Kovnat DM, Snider GL (1978) A prospective cooperative study of complications following flexible fiberoptic bronchoscopy. Chest 73: 813-816. [Crossref]

3. Kreider ME, Lipson DA (2003) Bronchoscopy for atelectasis in the ICU: a case report and review of the literature. Chest 124: 344-350. [Crossref]

4. Trouillet JL, Guiguet M, Gibert C, Fagon JY, Dreyfuss D, et al. (1990) Fiberoptic bronchoscopy in ventilated patients. Evaluation of cardiopulmonary risk under midazolam sedation. Chest 97: 927-933. [Crossref]
5. Hanson RR, Zavala DC, Rhodes ML, Keim LW, Smith JD (1976) Transbronchial biopsy via flexible fiberoptic bronchoscope; results in 164 patients. Am Rev Respir Dis 114: 67-72. [Crossref]

6. Da Conceiçao M, Genco G, Favier JC, Bidallier I, Pitti R (2000) Fiberoptic bronchoscopy during noninvasive positive-pressure ventilation in patients with chronic obstructive lung disease with hypoxemia and hypercapnia. Ann Fr Anesth Reanim 19: 231-236. [Crossref] 